\title{
Anomalous transport in holographic boundary conformal field theories
}

\author{
Chong-Sun $\mathrm{Chu}^{a, b}$ and Rong-Xin Miao ${ }^{a, c}$ \\ ${ }^{a}$ Physics Division, National Center for Theoretical Sciences, National Tsing-Hua University, \\ 101 Section 2 Kuang Fu Road, Hsinchu 30013, Taiwan \\ ${ }^{b}$ Department of Physics, National Tsing-Hua University, \\ 101 Section 2 Kuang Fu Road, Hsinchu 30013, Taiwan \\ ${ }^{c}$ School of Physics and Astronomy, Sun Yat-Sen University, \\ 2 Daxue Road, Zhuhai 519082, China \\ E-mail: cschu@phys.nthu.edu.tw, miaorongxin.physics@gmail.com
}

ABSTRACT: Recently, it is found that when an external magnetic field parallel to the boundary is applied, Weyl anomaly gives rises to a new anomalous current transport in the vicinity of the boundary. At the leading order of closeness from the boundary, the current is determined universally by the central charge of the theory. In this paper, we give a holographic proof for the existence and universality for this transport phenomena. We show that the current is independent of boundary conditions in four dimensions while it depends on boundary conditions in other dimensions. We also study the backreaction of the bulk Maxwell fields on the AdS spacetime and obtain the holographic Weyl anomaly for 5d BCFTs in presence of the background field strength.

KEywords: AdS-CFT Correspondence, Anomalies in Field and String Theories

ARXiv EPrint: 1804.01648 


\section{Contents}

1 Introduction 1

2 Holographic current for 4d BCFT 3

3 Holographic current in higher dimensions 5

$\begin{array}{lll}4 & \text { Holographic Weyl anomaly } & 7\end{array}$

5 Conclusions and discussions $\quad 10$

\section{Introduction}

The quantum transportation of charges induced by anomaly is an interesting phenomenon. ${ }^{1}$ For example, the chiral magnetic effect (CME) [3-7] refers to the generation of currents parallel to an external magnetic field. And the chiral vortical effect (CVE) [8-14] refers to the generation of a current due to rotational motion in the charged fluid. Recently, it has also been pointed out that anomalous transport also occurs in a conformally flat gravitational spacetime due to Weyl anomaly $[15,16]$. It should be noted that these kinds of anomalous transport occurs only in a material system where the chemical potentials are non-vanishing, or in a curved spacetime. As anomaly is an intrinsic property of the vacuum, it is interesting to ask if anomalous current transport could be realized in vacuum without the presence of material system.

One of the most well known manifestation of the quantum nature of the vacuum is the Casimir effect [17-19]. This occurs since the energy of the vacuum is sensitive to the change in the boundary condition. Recently the Casimir effect has been analyzed in full generality for arbitrary shape of boundary and for arbitrary spacetime metric, and universal relations between the Casimir coefficients and the boundary central charge in a boundary conformal field theory (BCFT) have been discovered [20]. The study was based on a field theory analysis of the properties of the energy momentum tensor in the vicinity of boundary. In a recent paper [21], we generalized the analysis to boundary system with $U(1)$ symmetry and discovered a new type of anomalous current in the vicinity of the boundary due to Weyl anomaly. ${ }^{2}$ In four dimensions, it was found that a current is induced near the boundary due to the presence of a background field strength

$$
J_{a}=\frac{4 b_{1} F_{a n}}{x}, \quad x \sim 0, \quad a=0,1,2,3
$$

\footnotetext{
${ }^{1}$ For a review see for example refs. [1, 2]

${ }^{2}$ Interestingly, [32] find that there is some evidence that non-Weyl anomalies such as 't Hooft anomalies are inconsistent with the existence of a boundary.
} 
Here $x$ is the geodesic distance from the boundary, $n$ is the direction of the inward pointing normal, and $b_{1}$ is the bulk central charge which appears in the Weyl anomaly [22] $]^{3}$

$$
\mathcal{A}=\int_{M} \sqrt{g}\left[b_{1} F_{\mu \nu} F^{\mu \nu}+\text { curvature terms }\right] .
$$

For the normalization of the gauge field kinetic term $S=-1 /\left(4 e^{2}\right) \int F^{2}, b_{1}$ is related to the beta function as $b_{1}=-\frac{\beta(e)}{2 e^{3}}$. Similar results were also found for higher dimensions where the anomalous current is determined universally by the central charge. We remark that the induced current (1.1) holds for not just boundary conformal field theories (BCFTs), but also for more general boundary quantum field theories (BQFTs) which are covariant, gauge invariant, unitary and renormalizable. Unlike the previous kinds of anomalous transport, this anomalous transport occurs in zero temperature vacuum in flat spacetime without the need of material support. And it is an intrinsic manifestation of the dependence of the quantum vacuum on boundary like the Casimir effect. Finally, it should be mentioned that there are boundary contributions to the current density which can exactly cancel the apparent "divergence" in the bulk current (1.1) at $x=0$ and define a finite total current [21].

BQFTs/BCFTs $[27,28]$ describe physical systems with boundaries. In recent years, the field of BCFTs has developed rapidly. In addition to traditional field theory techniques, see, e.g. [20, 29-36], holographic models of BCFTs have been developed in [20, 37-42] which allow for non-perturbative analysis of the boundary systems. Holographic dual of BCFT was originally introduced by Takayanagi [37] by considering a Neumann boundary condition in the bulk dual manifold. However the tensor type embedding equations of the proposal contain too many constraints in general and cannot be solved consistently for general shape of boundary. The difficulty was analyzed in $[40,41]$ and a consistent model of holographic BCFT was found by replacing the tensor type embedding equation by a scalar type embedding equation. More recently we found that [20] the original set of tensor embedding equations can also be consistent if one is to allow for a non-FG expansion of the metric in the bulk.

The models [40,41] and [20] of holographic BCFT have been applied to study the one point function of stress tensor. Boundary Weyl anomaly as well as new universal relations between the generalized Casimir coefficients and the central charges have been obtained in both models, and the results agree exactly, except for a different representation of the central charges as functions of the holographic BCFT parameter. In this paper, we study the anomalous current transport in this two models of holographic BCFTs, and show that to the leading order of closeness to the boundary, the holographic current is determined universally by the central charges of the Weyl anomaly. We also show that the current is independent of boundary conditions in four dimensions while it depends on boundary conditions in higher dimensions. The results agree with those obtained in [21] and generalize

\footnotetext{
${ }^{3}$ For parity-odd theory, it is possible that the Weyl anomaly includes one additional term [23-25] $\Delta \mathcal{A}=$ $b_{2} \int_{M} \sqrt{g} \epsilon^{i j k l} F_{i j} F_{k l}$. This is just a total derivative term and gives the Chern-Simons action on the boundary after the integral. However it is controversial whether such terms are allowed [26]. Even such terms are allowed, [25] notice that the coefficients $b_{2}$ are imaginary, which would violate the unitarity. Thus for unitary QFT we are interested of, the most general form of Weyl anomaly is given by (1.2).
} 


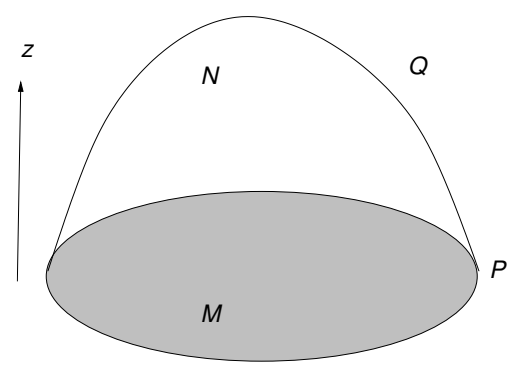

Figure 1. BCFT on $M$ and its dual $N$.

to theories that do not necessary admit a Lagrangian formulation. That the two proposals of holographic models yield again the same results for the anomalous current. This confirms our previous speculation that the two models of holographic BCFT correspond to two different kinds of BCFT which admit different holographic descriptions [20].

The paper is organized as follows. In section 2, we derive the holographic current for $4 \mathrm{~d}$ BCFTs and show that it is independent of boundary conditions and is determined entirely by the bulk central charge. In section 3, we generalize our discussions to higher dimensions and show that the holographic current depends on boundary conditions in higher dimensions. In section 4, we study the back reactions of Maxwell's fields to AdS spacetime and derive the holographic Weyl anomaly for 5d BCFTs. The paper is ended with some future discussions in section 5 .

\section{Holographic current for 4d BCFT}

Consider a boundary conformal field theory (BCFT) defined on a manifold $M$ with a boundary $P$. Takayanagi [37] proposed to extend the $d$ dimensional manifold $M$ to a $(d+1)$ dimensional asymptotically AdS space $N$ such that $\partial N=M \cup Q$, where $Q$ is a $d$ dimensional manifold with boundary $\partial Q=\partial M=P$. See figure 1 .

To investigate the renormalized current in holographic models of BCFT, let us add a $\mathrm{U}(1)$ gauge field to the holographic model. Following [37-39], we consider the following gauge invariant action for holographic BCFT $\left(16 \pi G_{N}=1\right)$

$$
I=\int_{N} \sqrt{G}\left(R-2 \Lambda-\frac{1}{4} \mathcal{F}_{\mu \nu} \mathcal{F}^{\mu \nu}\right)+2 \int_{Q} \sqrt{\gamma}(K-T) .
$$

Here $\mathcal{F}$ is the bulk field strength which reduces to $F$ on the boundary $M$. The constant parameter $T$ can be regarded as the holographic dual of boundary conditions of BCFT [40, 41]. The central issue in the construction of the AdS/BCFT is the determination of the location of $Q$ in the bulk. Takayanagi [37] proposed to impose the Neumann boundary condition (NBC) on $Q$. In our case this means we have on $Q$ :

$$
\begin{aligned}
K_{\alpha \beta}-(K-T) \gamma_{\alpha \beta} & =0, \\
\mathcal{F}_{\mu \nu} n_{Q}^{\mu} \Pi^{\nu}{ }_{\alpha} & =0,
\end{aligned}
$$

where $n_{Q}$ is the inward-pointing normal vector on $Q$, and $\Pi$ is the projection operator which gives the vector field and metric on $Q: \bar{A}_{\alpha}=\Pi_{\alpha}^{\nu} \mathcal{A}_{\mu}$ and $\gamma_{\alpha \beta}=\Pi_{\alpha}^{\mu} \Pi_{\beta}^{\nu} G_{\mu \nu}$. As 
discussed in $[40,41]$, the tensor embedding equations (2.2) admit no solution in general if the bulk metric is assumed to admit a FG expansion. On the other hand, (2.2) becomes consistent if a non-FG expanded bulk metric is adopted. Another possible holographic model for BCFT $[40,41]$ is to consider a mixed boundary condition on the metric whereby one obtains the scalar embedding equation

$$
K=\frac{d}{d-1} T
$$

together with (2.3). This model is also consistent. Both models yield the same consistent results for the Weyl anomaly as well as giving the same universal relations between the generalized Casimir coefficients and the central charges, the later of which are however parametrized differently in terms of $T$ for the two holographic models. In the following, we will consider both proposals of holographic BCFT. Quite amazingly, we find again that both models give the same results for the induced current when the result is expressed in terms of the central charges. This is quite remarkable and is a reconfirmation of the earlier indication that the two proposals of holographic models corresponds to two different kinds of BCFT [20].

Now back to our case. For simplicity, let us consider the case of a flat half space $x \geq 0$. The bulk metric reads

$$
d s^{2}=\frac{d z^{2}+d x^{2}+\delta_{a b} d y^{a} d y^{b}}{z^{2}} .
$$

In this case, (2.2) reduces to (2.4), and $Q$ is given by [37]

$$
x=-\sinh \rho z,
$$

where we have re-parametrized $T=3 \tanh \rho$. As for the solution for the vector field, due to the planar symmetry of the boundary, we consider $A_{\mu}$ that depends only on the coordinates $z$ and $x$. The Maxwell equations $\nabla_{\mu} \mathcal{F}^{\mu \nu}=0$ can be solved with $\mathcal{A}_{z}=\mathcal{A}_{z}(z), \mathcal{A}_{x}=\mathcal{A}_{x}(x)$ and $\mathcal{A}_{a}$ satisfying,

$$
z \partial_{x}^{2} \mathcal{A}_{a}-\partial_{z} \mathcal{A}_{a}+z \partial_{z}^{2} \mathcal{A}_{a}=0
$$

One can solve the above equation by separation of variables $\mathcal{A}_{a}(z, x)=Z(z) X(x)$, and then substitute the general solutions to (2.3) to obtain the solution by brute force. However there is a quicker trick. Inspired by similar considerations in [20], let us take the following ansatz for the vector field

$$
\mathcal{A}_{a}=A_{a}^{(0)}+x f_{1}\left(\frac{z}{x}\right) A_{a}^{(1)}+x^{2} f_{2}\left(\frac{z}{x}\right) A_{a}^{(2)}+\cdots,
$$

where we set $f_{i}(0)=1$ so that $\mathcal{A}_{a}$ reduce to the guage field $A_{a}$ at the AdS boundary $z=0$. Here $A^{(i)}$ are the expansion coefficients of $A_{a}$ about the boundary:

$$
A_{a}=A_{a}^{(0)}+x A_{a}^{(1)}+\cdots .
$$

In particular, $A_{a}^{(1)}$ is given by the field strength at the boundary:

$$
A_{a}^{(1)}=F_{x a}=F_{n a} .
$$


Note that in the derivative expansions we have $O\left(A^{(i)}\right) \sim O(\partial)^{i}$. Substituting (2.8) into (2.7) we get

$$
s\left(s^{2}+1\right) f_{1}^{\prime \prime}(s)-f_{1}^{\prime}(s)=0,
$$

at the linear order $O(\partial)$. Recall that $f_{1}(0)=1$, we have the solution $f_{1}(s)=1-c_{1}+$ $c_{1} \sqrt{1+s^{2}}$, and (2.8) reads

$$
\mathcal{A}_{a}=A_{a}^{(0)}+\left(\left(1-c_{1}\right) x+c_{1} \sqrt{x^{2}+z^{2}}\right) A_{a}^{(1)},
$$

where we have ignored the higher order terms since they are irrelevant to the current (1.1) of order $O(\partial)$, or equivalently, $O(F)$. Note also that we have analytic continuated $x \sqrt{1+\frac{z^{2}}{x^{2}}}$ to $\sqrt{x^{2}+z^{2}}$ in order to get smooth solution at $x=0$. Imposing the boundary condition (2.3) on $Q$, we get $c_{1}=1$. One can check directly that the solution $\mathcal{A}_{z}=\mathcal{A}_{z}(z), \mathcal{A}_{x}=\mathcal{A}_{x}(x)$ and

$$
\mathcal{A}_{a}=A_{a}^{(0)}+A_{a}^{(1)} \sqrt{x^{2}+z^{2}}
$$

is indeed an exact solution to the Maxwell equations and the boundary condition (2.3) in AdS.

From the gravitational action (2.1), we can derive the holographic current as [43]

$$
\left\langle J^{a}\right\rangle=\lim _{z \rightarrow 0} \frac{\delta I}{\delta A_{a}}=\lim _{z \rightarrow 0} \sqrt{G} \mathcal{F}^{z a}
$$

Substituting the solutions (2.5), (2.13) into (2.14), we obtain

$$
\left\langle J_{a}\right\rangle=\left.\partial_{z}^{2} \mathcal{A}_{a}\right|_{z=0}=-\frac{F_{a n}}{x}+O(1),
$$

where we have used (2.10). On the other hand, the holographic Weyl anomaly of (2.1) is obtained in [44] with the central charge given by

$$
b_{1}=-\frac{1}{4} \text {. }
$$

Now it is clear that the holographic BCFT satisfies the universal law of current (1.1). It is remarkable that current $(2.15)$ is independent of the parameter $T$, which shows that near-boundary current for $4 \mathrm{~d}$ BCFT is indeed independent of boundary conditions.

\section{Holographic current in higher dimensions}

In this section, we study the holographic current for BCFTs in higher dimensions. We verify that the leading term of the current is determined universally by the central charge of Weyl anomaly. However unlike the $4 \mathrm{~d}$ case, the current depends on boundary conditions in higher dimensions.

We start with the gravitational action

$$
I=\int_{N} \sqrt{G}\left(R-2 \Lambda-\frac{1}{4} \mathcal{F}_{\mu \nu} \mathcal{F}^{\mu \nu}\right)+2 \int_{Q} \sqrt{\gamma}(K-T) .
$$


We work in AdS spacetime (2.5) with the bulk boundary $Q$ given by (2.6). Focus on the leading order of current, we can ignore the back reactions of the Maxwell's fields to spacetime and the location of the bulk boundary. For plane boundary, $A_{\mu}$ depends on only the coordinates $z$ and $x$. For general dimension $d$, the Maxwell's equations can be solved with $\mathcal{A}_{z}=\mathcal{A}_{z}(z), \mathcal{A}_{x}=\mathcal{A}_{x}(x)$ and

$$
z \partial_{x}^{2} \mathcal{A}_{a}-(d-3) \partial_{z} \mathcal{A}_{a}+z \partial_{z}^{2} \mathcal{A}_{a}=0
$$

Similarly, the boundary condition (2.3) becomes

$$
\left.\left(\partial_{x} \mathcal{A}_{a}+\sinh \rho \partial_{z} \mathcal{A}_{a}\right)\right|_{x=-z \sinh \rho}=0 .
$$

As before, we consider the ansatz for the gauge field

$$
\mathcal{A}_{a}=A_{a}^{(0)}+A_{a}^{(1)} x f\left(\frac{z}{x}\right),
$$

where $f(0)=1$ and $A_{a}^{(i)}$ are constants. The Maxwell's equations (3.2) becomes

$$
s\left(s^{2}+1\right) f^{\prime \prime}(s)-(d-3) f^{\prime}(s)=0 .
$$

It has the general solution

$$
f(s)=1+\alpha_{d} \frac{s^{d-2}{ }_{2} F_{1}\left(\frac{d-3}{2}, \frac{d-2}{2} ; \frac{d}{2} ;-s^{2}\right)}{d-2},
$$

where $\alpha_{d}$ is an integration constant. It should be mentioned that, in order to get regular solutions at $x=0$, suitable analytic continuation of the hypergeometric function should be taken when one express the above solutions in terms of the coordinates $z$ and $x$. With the ansatz (3.4), the boundary condition (3.3) is simplified to

$$
\cosh \rho \operatorname{coth} \rho f^{\prime}(-\operatorname{csch} \rho)+f(-\operatorname{csch} \rho)=0 .
$$

Substituting (3.6) into (3.7), we get the integration constant

$$
\alpha_{d}=\frac{(2-d) \operatorname{csch}^{3} \rho(-\operatorname{coth} \rho)^{d}}{\operatorname{csch} \rho_{2} F_{1}\left(\frac{d-3}{2}, \frac{d-2}{2} ; \frac{d}{2} ;-\operatorname{csch}^{2} \rho\right)(\operatorname{coth} \rho \operatorname{csch} \rho)^{d}+(d-2) \cosh \rho \operatorname{coth}^{4} \rho(-\operatorname{csch} \rho)^{d}} .
$$

Notice that suitable analytic continuation of the hypergeometric function should be taken in order to get smooth function at $\rho=0$. For example, we have for $d=4,5$,

$$
\alpha_{4}=1, \quad \alpha_{5}=\frac{2}{\pi+4 \tan ^{-1}\left(\tanh \left(\frac{\rho}{2}\right)\right)} .
$$

Now we are ready to derive the holographic current. Substituting (2.5), (3.4) and (3.6) into (2.14), we obtain

$$
\left\langle J_{a}\right\rangle=\lim _{z \rightarrow 0} \frac{\partial_{z} \mathcal{A}_{a}}{z^{d-3}}=-\alpha_{d} \frac{F_{a n}}{x^{d-3}}+O\left(\frac{1}{x^{d-4}}\right),
$$

which takes the correct near-boundary behavior [21] and agrees with the $4 \mathrm{~d}$ result (2.15). Note that $\alpha_{d}$ depends on $\rho$ for $d \geq 5$, which means that the near-boundary current depends on the boundary conditions in higher dimensions. According to [21], $\alpha_{d}$ is related to the central charge of Weyl anomaly. In the next section, we will show that, similar to the $4 \mathrm{~d}$ BCFTs, this is also the case for 5d BCFTs with gravity duals. 


\section{Holographic Weyl anomaly}

In this section, we calculate the holographic Weyl anomaly of 5d BCFTs. For our purpose, it is sufficient to focus on the flat space with a plane boundary. Then all the curvatures and extrinsic curvatures vanish and only the field strength contribute to Weyl anomaly. In this case the Weyl anomaly takes the form

$$
\mathcal{A}=\int_{\partial M} d x^{4} \sqrt{h}\left[b_{1} F_{n a} F^{n a}+b_{2} F_{a b} F^{a b}\right]
$$

where $b_{1}$ and $b_{2}$ are boundary central charges, which depend on BCs. In [21] we have derived the relation between the current coefficient and the boundary central charge

$$
\alpha_{5}=2 b_{1}
$$

that holds universally for any BCFT with a path integral formulation. Using our holographic Weyl anomaly derived below, we prove that the universal relation (4.2) is verified for any holographic BCFT.

According to [45], holographic Weyl anomaly can be obtained as the UV logarithmic divergent terms of the gravitational action (3.1). Since the Weyl anomaly (4.1) is of order $O\left(F^{2}\right)$, we need to solve for the metric up to order $O\left(F^{2}\right)$ and the gauge field up to order $O(F)$. This means that we have to take into account of the back reaction of the Maxwell's fields to the spacetime metric. The solutions of the Maxwell fields up to order $O(F)$ has been obtained in section 3. Without loss of generality, we set all components of the gauge field to be zero except $\mathcal{A}_{y_{1}}$ :

$$
\mathcal{A}_{\mu}=\left(0,0, \operatorname{Fxf}\left(\frac{z}{x}\right), 0,0\right)
$$

Here we have denoted $F:=F_{n y_{1}}$ and $f(s)$ is given by (3.6), (3.9). Inspired by [20], we consider the following ansatz of metric for $x>0$

$$
d s^{2}=\frac{1}{z^{2}}\left[d z^{2}+\left(1+F^{2} x^{4} X\left(\frac{z}{x}\right)\right) d x^{2}+\left(\delta_{a b}+F^{2} x^{4} Q_{a b}\left(\frac{z}{x}\right)\right) d y^{a} d y^{b}\right]+O\left(F^{3}\right)
$$

where $Q_{a b}(s)=\operatorname{diag}\left(Q_{1}(s), Q_{2}(s), Q_{3}(s), Q_{4}(s)\right)$. The solutions for $x<0$ can be obtained by analytic continuation. The ansatz (4.4) works well in odd dimensions. For even-dimensional BCFTs, we need to add extra terms to (4.4) due to the presence of bulk Weyl anomaly. We consider

$$
X(0)=0, \quad Q_{a b}(0)=0,
$$

so that the BCFT lives in a flat space. Solving Einstein-Maxwell equations up to order $O\left(F^{2}\right)$

$$
R_{\mu \nu}-\frac{R-2 \Lambda}{2} G_{\mu \nu}=\frac{1}{2}\left(\mathcal{F}_{\mu \rho} \mathcal{F}_{\nu}{ }^{\rho}-\frac{1}{4} \mathcal{F}_{\sigma \rho} \mathcal{F}^{\sigma \rho} G_{\mu \nu}\right)
$$


we obtain

$$
\begin{aligned}
96 X(s)= & 15 s^{4}+\alpha_{5}\left(34 s^{3}+6 s\right)+\alpha_{5}^{2}\left(\left(60 s^{2}+46\right) \log \left(s^{2}+1\right)-s^{2}\left(21 s^{2}+43\right)\right) \\
& -2 \alpha_{5}\left(\alpha_{5} s\left(17 s^{2}+3\right)+3\left(5 s^{4}+6 s^{2}+1\right)\right) g(s) \\
& +3 \alpha_{5}^{2}\left(5 s^{4}+6 s^{2}+1\right) g(s)^{2}, \\
384 Q_{a}(s)= & 12 s\left(5 s^{3}-4 \lambda_{a}\left(5 s^{2}+3\right)\right) \\
& -6 \alpha_{5} s\left(13 s^{2}+3\right)+\alpha_{5}^{2}\left(-96 s^{4}-109 s^{2}+40 \log \left(s^{2}+1\right)\right) \\
& +2\left(\alpha_{5}^{2}\left(139 s^{2}+69\right) s+72 \lambda_{a}\left(s^{2}+1\right)^{2}+\alpha_{5}\left(-39 s^{4}+42 s^{2}+9\right)\right) g(s) \\
& -3 \alpha_{5}^{2}\left(7 s^{4}+54 s^{2}+23\right) g(s)^{2}, \quad a=1,2,3, \\
384 Q_{4}(s)= & -12 s\left(3 s^{3}-4\left(\lambda_{1}+\lambda_{2}+\lambda_{3}\right)\left(5 s^{2}+3\right)\right) \\
& -\alpha_{5} s\left(38 s^{2}-6\right)+\alpha_{5}^{2}\left(48 s^{4}-s^{2}+40 \log \left(s^{2}+1\right)\right) \\
& +\left(\alpha_{5}\left(90 s^{4}+36 s^{2}-6\right)-2 \alpha_{5}^{2}\left(41 s^{2}+39\right) s-144\left(\lambda_{1}+\lambda_{2}+\lambda_{3}\right)\left(s^{2}+1\right)^{2}\right) g(s) \\
& +\alpha_{5}^{2}\left(-9 s^{4}+54 s^{2}+39\right) g(s)^{2},
\end{aligned}
$$

where $\lambda_{1}, \lambda_{2}, \lambda_{3}$ are integration constants, $s=z / x$ and $g(s)=\tan ^{-1}(s)$ for $x>0$. In order to get continuous solutions at $x=0$, one must perform suitable analytic continuation for $g(s)$. In this way, we get $g(x, z)=\frac{\pi}{2}-2 \tan ^{-1}\left(x /\left(z+\sqrt{z^{2}+x^{2}}\right)\right)$.

It should be mentioned that the back-reacted spacetime (4.4), (4.7) is well-defined and has no physical divergence at $x=0$. To see this, let us calculate the following geometric invariants. We have

$$
\begin{aligned}
\lim _{x \rightarrow 0} R & =-30+\frac{z^{4}}{16}\left(\left(4+\pi^{2}\right) \alpha_{5}^{2}-4 \pi \alpha_{5}+4\right) F^{2}+O\left(F^{3}\right) \\
\lim _{x \rightarrow 0} R_{\mu \nu} R^{\mu \nu} & =150-\frac{5 z^{4}}{8}\left(\left(4+\pi^{2}\right) \alpha_{5}^{2}-4 \pi \alpha_{5}+4\right) F^{2}+O\left(F^{3}\right) \\
\lim _{x \rightarrow 0} R_{\mu \nu \rho \sigma} R^{\mu \nu \rho \sigma} & =60-\frac{z^{4}}{4}\left(\left(4+\pi^{2}\right) \alpha_{5}^{2}-4 \pi \alpha_{5}+4\right) F^{2}+O\left(F^{3}\right),
\end{aligned}
$$

which are indeed finite at $x=0$. Note that the perturbation solutions work well only for small $F$. Strictly speaking, one can only define 'small' for a dimensionless number. Thus by 'small' we actually means $F x^{2} \ll 1$ and $F z^{2} \ll 1$ (Note that $x$ and $z$ can be large but $F x^{2}$ and $F z^{2}$ should keep small). From (4.8), (4.9), (4.10), it is clear that the back reactions to spacetime are not only finite and also small. Recall that we are interested in the nearboundary current. According to [21], by 'near' it means $x \ll 1 / \sqrt{F}$. Thus the perturbation solutions with $F x^{2} \ll 1$ are sufficient for our purpose. Finally, we want to mention that the back-reacted spacetime (4.4), (4.7) is asymptotically AdS in the sence that

$$
\lim _{z \rightarrow 0} R_{\rho \sigma}^{\mu \nu}=-\left(\delta_{\rho}^{\mu} \delta_{\sigma}^{\nu}-\delta_{\sigma}^{\mu} \delta_{\nu}^{\nu}\right)+O\left(F^{3}\right)
$$

where we have set the AdS radius $l=1$.

Following [20], we assume embedding function of $Q$ takes the form

$$
x=-\sinh (\rho) z+\lambda_{4} F^{2} z^{5}+O\left(F^{3}\right)
$$


where $\lambda_{4}$ is a function of $\rho$. This is to be solved using either of the boundary conditions (2.2) or (2.4), and define consistent model of holographic BCFT respectively. Let us first consider the boundary condition (2.2) on $Q$ [37]

$$
K_{\alpha \beta}-(K-T) \gamma_{\alpha \beta}=0 .
$$

In this case, the intergation constants are fixed by the extra equations in (4.13):

$$
\begin{aligned}
\lambda_{1}= & \frac{1}{6}\left(\alpha_{5}\left(2-3 \alpha_{5} p(\rho)\right)+3 \sinh (\rho)\left(\alpha_{5} p(\rho)+1\right)^{2}\right), \\
\lambda_{2}= & \lambda_{3}=\frac{1}{6}\left(\alpha_{5}\left(3 \alpha_{5} p(\rho)+2\right)-3 \sinh (\rho)\left(\alpha_{5} p(\rho)+1\right)^{2}\right), \\
\lambda_{4}= & \frac{\alpha_{5}(-40 \cosh (2 \rho)-15 \cosh (4 \rho)+87)+12(6 \sinh (\rho)+\sinh (3 \rho))}{3840} \\
& +\frac{\alpha_{5}^{2}\left(17 \sinh (\rho)-71 \sinh (3 \rho)+4 \sinh ^{3}(\rho)(43 \cosh (2 \rho)+57) \log \left(\operatorname{coth}^{2}(\rho)\right)\right)}{3840} \\
& -\frac{p(\rho)\left(\alpha_{5}\left(\alpha_{5}(40 \cosh (2 \rho)+15 \cosh (4 \rho)-87)-6(21 \sinh (\rho)+5 \sinh (3 \rho)) \cosh ^{2}(\rho)\right)\right)}{3840} \\
& +\frac{\alpha_{5}^{2} p(\rho)^{2}(21 \sinh (\rho)+5 \sinh (3 \rho)) \cosh ^{2}(\rho)}{1280},
\end{aligned}
$$

where $p(\rho):=-2 \tan ^{-1}\left(\frac{\sinh (\rho)}{\cosh (\rho)+1}\right)-\frac{\pi}{2}$ and $\alpha_{5}$ is given by (3.9). It is remarkable that the (4.13) determines not only the integration constants of the metric but also the location of bulk boundary $Q$. That is because we only need one equation to fix the codimension one surface $Q$, however there are many extra equations in the BC (4.13). The extra equations help to fix the bulk metric in addition to the location of $Q$.

Next let us consider the boundary condition (2.4). One can solve for $\lambda_{4}$ by substituting the embedding function (4.12) into (2.4). It turns out that $\lambda_{4}$ is unchanged and is given by exactly the same expression (4.14) with $\alpha_{5}$ given by (3.9). Note that unlike the BC (2.2), the $\mathrm{BC}(2.4)$ does not fix the integration constants $\left(\lambda_{1}, \lambda_{2}, \lambda_{3}\right)$ of the bulk metric (4.7). It is remarkable that, as we will show below, the Maxwell field strength part of the Weyl anomaly is independent of these parameters $\left(\lambda_{1}, \lambda_{2}, \lambda_{3}\right)$.

Now we have worked out the bulk metric and the embedding function of $Q$ up to order $O\left(F^{2}\right)$. Let us go on to calculate the holographic Weyl anomaly. We will keep the parameters $\left(\lambda_{1}, \lambda_{2}, \lambda_{3}\right)$ free so that our following discussion apply to both the BCs $(2.2)$ and (2.4). By using the EOM (4.6) and NBC (2.4), we can rewrite the on-shell gravitational action (3.1) as

$$
I=\int_{N} \sqrt{G}\left[-10-\frac{1}{8} \mathcal{F}_{\mu \nu} \mathcal{F}^{\mu \nu}\right]+2 \int_{Q} \sqrt{\gamma} \tanh \rho .
$$

To get the holographic Weyl anomaly, we need to do the integration along $x$ and $z$, and then select the UV logarithmic divergent terms. We divide the integration region into two parts: region I is defined by $(z \geq 0, x \geq 0)$ and region II is defined by the complement of region I. Let us first study the integration in region I, where only the bulk action in (4.15) 
contributes. Integrating along $z$ and then expanding the result in small $z=\epsilon_{z}$, we obtain

$$
\begin{aligned}
I_{1} & =\int_{\epsilon_{x}} d x\left[\frac{2 \alpha_{5}}{3 x} F_{n a} F^{n a}-\frac{13}{32 \epsilon_{z}} F_{n a} F^{n a}-\frac{2}{\epsilon_{z}^{5}}+O(1)\right] \\
& =\log \left(\frac{1}{\epsilon_{x}}\right) \frac{2 \alpha_{5}}{3} F_{n a} F^{n a}+\cdots .
\end{aligned}
$$

Next let us consider the integration in region II. In this case, both the bulk action and boundary action in (4.15) contribute. For the bulk action, we first do the integral along $x$, which yields a boundary term on $Q$. Note that since only the UV logarithmic divergent terms are related to Weyl anomaly, we keep only the lower limit of the integral of $x$. Adding the boundary term from bulk integral to the boundary action in (4.15), we obtain

$$
\begin{aligned}
I_{2} & =\int_{\epsilon_{z}} d z\left[-\frac{\alpha_{5}}{6 z} F_{n a} F^{n a}+\frac{2 \sinh (\rho)}{z^{5}}+O(1)\right] \\
& =\log \left(\frac{1}{\epsilon_{z}}\right) \frac{-\alpha_{5}}{6} F_{n a} F^{n a}+\cdots .
\end{aligned}
$$

Note that the results (4.16) and (4.17) are independent of $\lambda_{1}, \lambda_{2}, \lambda_{3}$. Note also that $\log \left(\frac{1}{\epsilon_{x}}\right)$ and $\log \left(\frac{1}{\epsilon_{z}}\right)$ are counted as the same divergence since they differ only by a finite term $\log l$ for $\epsilon_{z}=l \epsilon_{x}$. Therefore, combining together (4.16) and (4.17), we finally obtain the Weyl anomaly (4.18)

$$
\mathcal{A}=\int_{\partial M} \sqrt{h} \frac{\alpha_{5}}{2} F_{n a} F^{n a} d^{4} x
$$

Hence we obtain the universal relation (4.2).

We remark that we have $F_{a b}=0$ for the solution (4.3). To get the information of central charge $b_{4}$ in (4.1), we need to consider solutions with $y^{a}$ dependence. We leave this problem to future study.

\section{Conclusions and discussions}

In this paper, we have studied the anomalous current transport in holographic BCFTs. We have verified that the holographic current is determined universally by the central charge of the Weyl anomaly. To leading order of nearness to the boundary, the current independent of boundary conditions in four dimensions while it depends on boundary conditions in higher dimensions. The holographic results obtained here support the results obtained recently in [21]. We have also studied the back reaction of bulk Maxwell's fields to AdS spacetime and obtain the holographic Weyl anomaly for 5d BCFTs. It will be interesting to study Schwinger effect [46] near the boundary. With the help of the boundary, the magnetic field can separate the virtual particle pairs and turn them into real particles. Thus, it is expected that constant magnetic field can produce non-trivial Schwinger effect in the vicinity of the boundary. We leave this problem for future study. 


\section{Acknowledgments}

We thank Dimitrios Giataganas, Bei-Lok Hu, Ling-Yan Hung, Yan Liu, Jian-Xin Lu and Tadashi Takayanagi for useful discussions and comments. This work is supported in part by NCTS and the grant MOST 105-2811-M-007-021 of the Ministry of Science and Technology of Taiwan.

Open Access. This article is distributed under the terms of the Creative Commons Attribution License (CC-BY 4.0), which permits any use, distribution and reproduction in any medium, provided the original author(s) and source are credited.

\section{References}

[1] D.E. Kharzeev, The chiral magnetic effect and anomaly-induced transport, Prog. Part. Nucl. Phys. 75 (2014) 133 [arXiv:1312.3348] [INSPIRE].

[2] K. Landsteiner, Notes on anomaly induced transport, Acta Phys. Polon. B 47 (2016) 2617 [arXiv: 1610.04413] [INSPIRE].

[3] A. Vilenkin, Parity nonconservation and neutrino transport in magnetic fields, Astrophys. J. 451 (1995) 700 [INSPIRE].

[4] A. Vilenkin, Equilibrium parity violating current in a magnetic field, Phys. Rev. D 22 (1980) 3080 [INSPIRE].

[5] M. Giovannini and M.E. Shaposhnikov, Primordial hypermagnetic fields and triangle anomaly, Phys. Rev. D 57 (1998) 2186 [hep-ph/9710234] [INSPIRE].

[6] A. Yu. Alekseev, V.V. Cheianov and J. Fröhlich, Universality of transport properties in equilibrium, Goldstone theorem and chiral anomaly, Phys. Rev. Lett. 81 (1998) 3503 [cond-mat/9803346] [INSPIRE].

[7] K. Fukushima, Views of the chiral magnetic effect, Lect. Notes Phys. 871 (2013) 241 [arXiv: 1209.5064] [INSPIRE].

[8] D. Kharzeev and A. Zhitnitsky, Charge separation induced by P-odd bubbles in QCD matter, Nucl. Phys. A 797 (2007) 67 [arXiv:0706.1026] [INSPIRE].

[9] J. Erdmenger, M. Haack, M. Kaminski and A. Yarom, Fluid dynamics of R-charged black holes, JHEP 01 (2009) 055 [arXiv:0809.2488] [INSPIRE].

[10] N. Banerjee, J. Bhattacharya, S. Bhattacharyya, S. Dutta, R. Loganayagam and P. Surowka, Hydrodynamics from charged black branes, JHEP 01 (2011) 094 [arXiv:0809.2596] [INSPIRE].

[11] D.T. Son and P. Surowka, Hydrodynamics with triangle anomalies, Phys. Rev. Lett. 103 (2009) 191601 [arXiv:0906.5044] [inSPIRE].

[12] K. Landsteiner, E. Megias and F. Pena-Benitez, Gravitational anomaly and transport, Phys. Rev. Lett. 107 (2011) 021601 [arXiv:1103.5006] [INSPIRE].

[13] S. Golkar and D.T. Son, (Non)-renormalization of the chiral vortical effect coefficient, JHEP 02 (2015) 169 [arXiv:1207.5806] [INSPIRE].

[14] K. Jensen, R. Loganayagam and A. Yarom, Thermodynamics, gravitational anomalies and cones, JHEP 02 (2013) 088 [arXiv: 1207.5824] [INSPIRE]. 
[15] M.N. Chernodub, Anomalous transport due to the conformal anomaly, Phys. Rev. Lett. 117 (2016) 141601 [arXiv: 1603.07993] [INSPIRE].

[16] M.N. Chernodub, A. Cortijo and M.A.H. Vozmediano, Generation of a Nernst current from the conformal anomaly in Dirac and Weyl semimetals, Phys. Rev. Lett. 120 (2018) 206601 [arXiv: 1712.05386] [INSPIRE].

[17] H.B.G. Casimir, On the attraction between two perfectly conducting plates, Indag. Math. 10 (1948) 261 [Kon. Ned. Akad. Wetensch. Proc. 51 (1948) 793] [Front. Phys. 65 (1987) 342] [Kon. Ned. Akad. Wetensch. Proc. 100N3-4 (1997) 61] [InSPIRE].

[18] G. Plunien, B. Müller and W. Greiner, The Casimir effect, Phys. Rept. 134 (1986) 87 [INSPIRE].

[19] M. Bordag, U. Mohideen and V.M. Mostepanenko, New developments in the Casimir effect, Phys. Rept. 353 (2001) 1 [quant-ph/0106045] [INSPIRE].

[20] R.-X. Miao and C.-S. Chu, Universality for shape dependence of Casimir effects from Weyl anomaly, JHEP 03 (2018) 046 [arXiv:1706.09652] [INSPIRE].

[21] C.-S. Chu and R.-X. Miao, Anomaly induced transport in boundary quantum field theories, arXiv: 1803.03068 [INSPIRE].

[22] M.J. Duff, Twenty years of the Weyl anomaly, Class. Quant. Grav. 11 (1994) 1387 [hep-th/9308075] [INSPIRE].

[23] M.J. Duff, Ultraviolet divergences in extended supergravity, in First school on supergravity, Trieste, Italy, 22 April-6 May 1981 [arXiv:1201.0386] [INSPIRE].

[24] Y. Nakayama, CP-violating CFT and trace anomaly, Nucl. Phys. B 859 (2012) 288 [arXiv:1201.3428] [INSPIRE].

[25] L. Bonora, S. Giaccari and B. Lima de Souza, Trace anomalies in chiral theories revisited, JHEP 07 (2014) 117 [arXiv: 1403.2606] [INSPIRE].

[26] F. Bastianelli and R. Martelli, On the trace anomaly of a Weyl fermion, JHEP 11 (2016) 178 [arXiv: 1610.02304] [INSPIRE].

[27] J.L. Cardy, Boundary conformal field theory, hep-th/0411189 [INSPIRE].

[28] D.M. McAvity and H. Osborn, Energy momentum tensor in conformal field theories near a boundary, Nucl. Phys. B 406 (1993) 655 [hep-th/9302068] [INSPIRE].

[29] D. Fursaev, Conformal anomalies of CFT's with boundaries, JHEP 12 (2015) 112 [arXiv: 1510.01427] [INSPIRE].

[30] C.P. Herzog, K.-W. Huang and K. Jensen, Universal entanglement and boundary geometry in conformal field theory, JHEP 01 (2016) 162 [arXiv:1510.00021] [INSPIRE].

[31] C. Herzog, K.-W. Huang and K. Jensen, Displacement operators and constraints on boundary central charges, Phys. Rev. Lett. 120 (2018) 021601 [arXiv: 1709.07431] [INSPIRE].

[32] K. Jensen, E. Shaverin and A. Yarom, 't Hooft anomalies and boundaries, JHEP 01 (2018) 085 [arXiv: 1710.07299] [INSPIRE].

[33] M. Kurkov and D. Vassilevich, Parity anomaly in four dimensions, Phys. Rev. D 96 (2017) 025011 [arXiv: 1704.06736] [INSPIRE].

[34] M. Kurkov and D. Vassilevich, Gravitational parity anomaly with and without boundaries, JHEP 03 (2018) 072 [arXiv: 1801.02049] [INSPIRE]. 
[35] D. Rodriguez-Gomez and J.G. Russo, Free energy and boundary anomalies on $\mathbb{S}^{a} \times \mathbb{H}^{b}$ spaces, JHEP 10 (2017) 084 [arXiv: 1708.00305] [INSPIRE].

[36] D. Seminara, J. Sisti and E. Tonni, Corner contributions to holographic entanglement entropy in $A d S_{4} / B C F T_{3}, J H E P 11$ (2017) 076 [arXiv: 1708.05080] [INSPIRE].

[37] T. Takayanagi, Holographic dual of BCFT, Phys. Rev. Lett. 107 (2011) 101602 [arXiv: 1105.5165] [INSPIRE].

[38] M. Fujita, T. Takayanagi and E. Tonni, Aspects of AdS/BCFT, JHEP 11 (2011) 043 [arXiv: 1108.5152] [INSPIRE].

[39] M. Nozaki, T. Takayanagi and T. Ugajin, Central charges for BCFTs and holography, JHEP 06 (2012) 066 [arXiv: 1205.1573] [INSPIRE].

[40] R.-X. Miao, C.-S. Chu and W.-Z. Guo, New proposal for a holographic boundary conformal field theory, Phys. Rev. D 96 (2017) 046005 [arXiv:1701.04275] [InSPIRE].

[41] C.-S. Chu, R.-X. Miao and W.-Z. Guo, On new proposal for holographic BCFT, JHEP 04 (2017) 089 [arXiv: 1701.07202] [INSPIRE].

[42] A. Faraji Astaneh and S.N. Solodukhin, Holographic calculation of boundary terms in conformal anomaly, Phys. Lett. B 769 (2017) 25 [arXiv:1702.00566] [INSPIRE].

[43] A. Gynther, K. Landsteiner, F. Pena-Benitez and A. Rebhan, Holographic anomalous conductivities and the chiral magnetic effect, JHEP 02 (2011) 110 [arXiv:1005.2587] [INSPIRE].

[44] P. Benetti Genolini, D. Cassani, D. Martelli and J. Sparks, Holographic renormalization and supersymmetry, JHEP 02 (2017) 132 [arXiv:1612.06761] [INSPIRE].

[45] M. Henningson and K. Skenderis, The holographic Weyl anomaly, JHEP 07 (1998) 023 [hep-th/9806087] [INSPIRE].

[46] J.S. Schwinger, On gauge invariance and vacuum polarization, Phys. Rev. 82 (1951) 664 [INSPIRE]. 